\title{
HECHOS RELEVANTES DE LA EDUCACIÓN EN EL SALVADOR (1821-1940)
}

\section{RELEVANT FACTS OF EDUCATION IN EL SALVADOR (1821-1940)}

Referencia del ensayo

Fuentes Cabrera, J. P. (2022). Hechos relevantes de la educación en el salvador (1821-1940) . Revista Científica del Sistema de Estudios de Postgrado de la Universidad de San Carlos de Guatemala, 5(1), 33-38. DOI: https://doi.org/10.36958/sep.v5i1.94

\author{
Jorge Pastor Fuentes Cabrera \\ jorge.fuentes@ues.edu.sv \\ https://orcid.org/0000-0002-5396-0668 \\ Doctorado en Educación con especialidad en Educación Superior \\ Universidad de El Salvador
}

Fecha de recibido: $31 / 10 / 2021$

Fecha de aceptado: 05/01/2022

\section{RESUMEN}

OBJETIVO: abordar aspectos relevantes de la educación en El Salvador, mediante un análisis de los hechos históricos ocurridos entre 1821 y 1940. MÉTODO: se realizó la revisión de los archivos digitales del Diario Oficial, así como también de otros documentos que contienen información relacionada con el tema en cuestión. RESULTADOS: para su estudio se han considerado tres momentos: la independencia de Centroamérica, la separación de la Federación Centroamericana y el triunfo de los liberales sobre los conservadores. CONCLUSIÓN: esta consideración permitió concluir que, la posición que se asume en relación a la educación de los habitantes de un país está determinada por las condiciones predominantes del contexto.

\section{PALABRAS CLAVE}

Educación, hechos históricos, liberales y conservadores

\section{ABSTRACT}

OBJECTIVE: the relevant facts of education in El Salvador; through an analysis of the historical events that occurred between 1821 and 1940. METHOD: the files of the Official Gazette were reviewed, as well as other documents containing information related to the subject in question. RESULTS: for its analysis, three moments have been considered: the independence of Central America, the separation of the Central American Federation and the triumph of the liberals over the conservatives. CONCLUSION: this consideration concludes that the position assumed in relation to the education of the inhabitants of a country is determined by the prevailing conditions of the context.

\section{KEYWORDS}

Education, historical facts, liberals and conservatives 


\section{INTRODUCCIÓN}

La forma de concebir la educación ha cambiado a lo largo de la historia. En El Salvador desde que fue declarada la independencia hasta el año 1940 ocurrieron importantes avances a diferentes ritmos de desarrollo; sin embargo, no ha sido un proceso sistemático, ni planificado. Muchas veces ha ocurrido como resultado de iniciativas individuales de los funcionarios de turno, a la visión de futuro de los gobernantes y a su interés por incorporar prácticas educativas consideradas exitosas a la escuela salvadoreña.

En el periodo incluido en el estudio, fácilmente se pueden identificar tres momentos; el primero caracterizado por mantener las influencias de la época colonial y el reacomodo natural que sufrió la república después de ser declarada la independencia de Centroamérica, el segundo ocurrido posterior a la separación de la Federación Centroamericana y el tercero marcado por el triunfo de los liberales sobre los conservadores. En cada uno de estos momentos se incorporan ideas aportadas por las generaciones anteriores, pero agregando elementos nuevos que en su momento representaron una innovación.

En El Salvador existe muy poca información relacionada con la dinámica que ha seguido el proceso docente educativo desde los niveles inferiores hasta el superior. Lo que aquí se presenta es una aproximación de manera general a las principales decisiones que fueron tomadas por las autoridades de turno; que tenían como fin principal realizar regulaciones en relación al funcionamiento administrativo, la infraestructura, la formación que reciben los profesores, el aprendizaje del alumno, los objetivos de la educación, las formas organizativas, los medios utilizados, los métodos elegidos, los contenidos a desarrollar y las estrategias evaluativas utilizadas en la escuela primaria.
Por lo anterior, en este artículo se pretende presentar los resultados obtenidos de la revisión teórica realizada de los archivos digitales del Diario Oficial, así como también de otros documentos que contienen información de la educación en El Salvador.

\section{CONTENIDO}

\section{La independencia de Centroamérica}

La escasa información existente sobre temas relacionados con la educación formal en los años posteriores a que se proclamara la independencia de Centroamérica y se estableciera la República Federal Centroamericana, hacen pensar que no fue un tema prioritario para los gobernantes de esa época. Según lo expresado por Segovia (2012), en la redacción de la Constitución Federal existen pocas referencias sobre regulaciones al funcionamiento de las instituciones educativas de ese tiempo. Fue hasta el año 1932, según lo refiere Aguilar Avilés como se cita en Segovia, (2012), que se crea el Primer Reglamento de la Enseñanza Primaria. Además, en este período aparecen informes relacionados con el método de enseñanza que debía ser aplicado, el Método Lancasteriano.

El método Lancasteriano fue creado por Joseph Lancaster, quien abrió en 1798 una escuela en el Barrio de Sulhwark de Londres. En grupos de diez un monitor, enseñaba a los demás niños y practicaban la lectura, con un éxito demostrable en Europa y en países de Latinoamérica. Debido a estos antecedentes fue adoptado en El Salvador para cumplir el objetivo de masificar la educación, tomando en cuenta que para ese tiempo habían pocos maestros. Para introducir esta forma de enseñanza según el Ministerio de Educación MINED (2009), el gobierno decidió contratar al educador brasileño José Coelho. 


\section{Separación de la Federación Centroa-} mericana

La inestabilidad política de ese momento trajo como resultado final la división de la Federación Centroamericana en el año 1841. La separación de El Salvador está registrada en el decreto publicado en la Gaceta de El Salvador el 19 de marzo de 1859. Este hecho permitió que se tomaran las decisiones importantes; en relación a la educación, por ejemplo, Avilés como se cita en Elizondo (2012), registra la creación el 13 de marzo de 1841 de cargos y organismos encargados de administrar la educación del país.

En este contexto según el MINED (2009), ocurre un suceso trascendental que marcó el inicio de la educación superior para el país. Por decreto de la Asamblea Constituyente se funda la Universidad de El Salvador el 16 de febrero de 1841. En los primeros años de existencia para la Universidad de El Salvador era difícil encontrar profesores nacionales y la solución fue traerlos desde Europa. Estos profesores después de un corto tiempo decidían irse del país generando dificultades para atender a los estudiantes. Además, otros datos significativos en este periodo fueron el apoyo a la educación de la mujer y el incremento progresivo de los estudiantes en las escuelas.

Cabe señalar, que en los 40 años posteriores a la independencia existen muy pocas decisiones en el campo educativo. Probablemente debido a la constante inestabilidad política; en este momento de la historia se dio la lucha entre liberales y conservadores. Durante el régimen del General Gerardo Barrios presidente de la República (1861-1863), con un gobierno de corte progresista, preocupado por la educación se crea el Reglamento de escuelas de primeras letras, publicado en el Diario Oficial del 6 de noviembre de 1861.
Allí se determinaba que en las escuelas se enseñaría la lectura, la escritura, la aritmética práctica, la ortografía y la doctrina cristiana. El método utilizado en la enseñanza fue el Lancasteriano, a pesar de las críticas recibidas por favorecer el aprendizaje memorístico.

\section{El triunfo de los liberales sobre los con- servadores}

La confrontación entre liberales y conservadores, según el MINED (2009), llegó a su final en el 1871, año en que se da el derrocamiento de Francisco Dueñas. Este acontecimiento marca el triunfo de los liberales y con ello la implementación de reformas relacionadas con el comercio, la influencia de la Iglesia en el estado entre otras cosas.

Las transformaciones según el MINED (2009), fueron evidentes fundamentalmente hacia 1870 y 1880 , se incorporan iniciativas que favorecen la educación de la mujer en áreas distintas a las relacionadas con aspectos del hogar. En el gobierno de (Mariscal Santiago González 1872), según Aguilar como se cita en Elizondo, (2012) se prioriza que los fines de la enseñanza estuvieran alrededor de la educación integral, dándole prioridad a la educación primaria y se estableció el funcionamiento de las escuelas, la enseñanza, funciones de maestros, directores y subdirectores.

En el proyecto sobre la reforma de la instrucción primaria publicado en el Diario Oficial el 3 de julio de 1885 se describen las causas que se han opuesto a la enseñanza. Las que se detallan siguen vigentes hasta hoy en día, continuamente se sigue hablando de la falta de idoneidad de los profesores, problemas en aplicar el método apropiado, la doble ocupación del maestro, la inasistencia a las escuelas, entre otros. Estos problemas aún siguen esperando ser resueltos y constituyen verdaderas trabas en el sistema educativo. 
En la presidencia del General Francisco Menéndez (1885-1890), se establecieron cambios importantes en la forma predominante de enseñanza de la época, la abolición del método de enseñanza Lancasteriano. En la memoria publicada en el Diario Oficial el 13 de mayo de 1889 , se argumenta que se rompió con el sistema antiguo que atormentaba el cuerpo y la memoria de los niños, con poco provecho para el corazón y la inteligencia y se sustituyó por el sistema de Pestalozzi.

Por otra parte, uno de los objetivos que se buscaba con las escuelas normales era dar soporte a las medidas adoptadas. Esto se logra ver en la memoria publicada en el Diario Oficial el 13 de mayo de 1889 . Se intentaba dotar a los profesores con nuevas herramientas pedagógicas. Era evidente que para el nuevo sistema se necesitaban muy buenos pedagogos. El gobierno lo comprendió así y organizó los colegios normales uno de varones y otro de señoritas para la formación de maestros.

En el Reglamento de Educación Pública Primaria, artículos 48 y 60 publicado en el Diario Oficial el 11 de junio 1889, describe las condiciones generales que debería tener el edificio de la escuela. Se pretendía garantizar en lo posible el mejor ambiente para la enseñanza con espacios físicos limpios, amplios y seguros para profesores y estudiantes. Queda establecido claramente la necesidad de aplicar los métodos más apropiados para asegurar una educación para la vida.

Debe señalarse, que en ocasiones se proponían modificaciones, pero no se ejecutaban; con fecha 25 de agosto de 1898 se decretó El Reglamento de Enseñanza Secundaria Moderna, que modifica el expedido el 15 de noviembre de 1896 . Se puede constatar en la Memoria de los Actos del Poder Ejecutivo en los ramos de la Instrucción Pública que se encuentra publicada en el Diario Oficial del día 11 de marzo de 1899; que el Gobierno
Provisional dispone poner en vigencia nuevamente el Reglamento de 1896.

Finalmente, la Secretaría Instrucción Pública en el Diario Oficial, Tomo No. 127, No. 267 del 8 de diciembre de 1939, publicó el decreto que contiene el plan y programas de enseñanza primaria urbana de El Salvador a regir desde el año de 1940. En dicho decreto encontramos consideraciones generales, los objetivos, las características (continuidad y correlación), propósitos, centro de observación y orientaciones para el desarrollo de la clase. Este dato hace evidente una mejor estructuración si se compara con los programas de años precedentes.

\section{CONCLUSIÓN}

Los inicios de la vida independiente de la República Federal Centroamericana estuvieron marcados por las luchas políticas entre centralistas y federalistas, lo que provocó que la educación de la población no fuera el principal tema de interés. Una década después se divulgó el Método Lancasteriano y se crea el Primer Reglamento de la Enseñanza Primaria, representando estas las primeras decisiones importantes en relación a la educación. El método Lancasteriano para 1832 se había constituido en una tendencia metodológica en los países latinoamericanos, como la solución rápida ante los altos niveles de analfabetismo que existía en ese momento; representaba la solución a la falta de maestros y las crecientes demandas educativas de la población.

Los problemas entre confederados y republicanos frenaron las posibilidades de desarrollo de la naciente república, provocando que los pocos recursos fueran dirigidos a financiar el ejército y se descuidaron áreas prioritarias como la educación. El fin de la Federación Centroamericana representó la oportunidad de tomar decisiones importantes. En este 
contexto se funda la Universidad de El Salvador y el comienzo del apoyo a la educación de la mujer con la creación del primer centro educativo para mujeres, derecho que había sido negado por muchos años.

Posteriormente, la lucha que se daba entre centralistas y federalistas, pasa a la pugna entre los liberales y conservadores, caracterizada por los constantes golpes de estado y gobiernos provisionales, que no generaba el ambiente propicio para el desarrollo. El triunfo de los liberales representó la posibilidad de satisfacer las aspiraciones de libertad, progreso económico y mayores niveles educativos; sin embargo, a pesar de existir logros concretos, estos no ocurrieron de forma inmediata o se continuaron utilizando las viejas estrategias para enfrentar los problemas.

La educación que por años estuvo bajo la tutela de la iglesia católica pasa a ser laica, en un período donde las posiciones liberales se hicieron indiscutibles. Evidentemente, no se buscaba eliminar a Dios de las escuelas; lo que se procuraba era que existiera tolerancia de cultos y que la religión no ocupará el centro en la formación del estudiante.

Las escuelas normales fueron utilizadas como estrategia para incidir en el cambio de metodología de enseñanza en las escuelas. La preparación de los maestros se esperaba que permitiera hacer la transición del método de Lancaster eminentemente memorístico al método de Pestalozzi caracterizado por propiciar el aprendizaje mediante la participación activa del estudiante. El papel del maestro era determinante en las escuelas, se consideraba como un elemento importante para elevar la calidad de la educación. Sin embargo, los profesores muchas veces no tenían las competencias que la sociedad exigía. Las razones que se han opuesto a la enseñanza siguen siendo las mismas aun en este tiempo. Las reformas realizadas encaminadas a intro- ducir mejoras a la educación salvadoreña se caracterizaron por el interés de dotar de más profesores al sistema educativo, mejorar la infraestructura de las escuelas, proporcionar el equipo necesario e incorporar nuevos contenidos a los planes de estudios.

En la adopción de los métodos de enseñanza ha predominado el éxito que su aplicación ha tenido en otras regiones, como los países de Europa o en Estados Unidos; con características evidentemente diferente a las de El Salvador, no se han considerado otras variables que debieran asegurarse para garantizar su éxito. Se tenía una concepción moderna sobre los métodos de enseñanza, se proponían aquellos que favorecieran la creatividad y el aprendizaje activo en los alumnos, el desarrollo de habilidades, formación de valores, etcétera, aplicables al contexto donde los estudiantes se desenvuelven. Evidentemente el sistema educativo se ha ido perfeccionando, pero aun falta mucho por hacer.

\section{REFERENCIAS}

Decreto declarando república libre, soberana e independiente, al estado de El Salvador. (19 de marzo de 1859). Diario oficial. 88. https://www. diariooficial.gob. sv/diarios/1859/1859_Parte4.pdf

Elizondo, L. (2012). Historia de la educación en El Salvador 1960-1989. Tesis de licenciatura en historia. Universidad de El Salvador. Tesis. https://ri.ues.edu. sv/id/eprint/2978/1/Historia\%20de\%20 la\%20Administraci\%C3\%B3n\%20de $\% 20$ la\%20Educaci\%C3\%B3n\%20en\%20El\%20 Salvador\%201960-1989.pdf

Memoria de los actos del poder ejecutivo en los ramos de la instrucción pública. (11 de marzo de 1899). Diario oficial. 455. https: / / www.diariooficial.gob.sv/diarios/1899/1899_Parte10.pdf 
Memoria presentada a la honorable asamblea nacional por el señor secretario de estado en los derechos de instrucción pública. (13 de mayo de 1889). Diario oficial. 573. https://www.diariooficial.gob.sv/ diarios/1889/1889_Parte16.pdf

Ministerio de Educación. (2009). Historia El Salvador 1. MINED. https://www.mined.gob.sv/descarga/cipotes/historia_ESA_Tomol_0_.pdf

Plan y programas de enseñanza primaria urbana de El Salvador. (8 de diciembre de 1939). Diario Oficial. 3771. https: //www. diariooficial.gob.sv/diarios/1939/19392T/1939-2T_Parte45.pdf

Proyecto sobre reforma de la instrucción primaria. (3 de Julio de 1885). Diario oficial. 9. https://www. diariooficial.gob. sv/diarios/1885/1885_Parte1.pdf

Reglamento de educación pública primaria. (11 de junio de 1889). Diario oficial. 697. https://www.diariooficial.gob.sv/ diarios/1889/1889_Parte19.pdf

Reglamento de las primeras letras. (6 de noviembre de 1861). Diario oficial. 1. https: / / www.diariooficial.gob.sv/diarios/1889/1889_Parte19.pdf
Segovia, D. (2012). Historia de la Administración de la Educación 1908-1960. Tesis de licenciatura en historia. Universidad de El Salvador. Tesis. https://ri.ues.edu.sv/ id/eprint/18113/1/14102207.pdf

\section{SOBRE EL AUTOR}

\section{Jorge Pastor Fuentes Cabrera}

Es Licenciado en Anestesiología e Inhaloterapia, graduado de la Maestría en Métodos y Técnicas de Investigación Social y actualmente estudia el Doctorado en Educación de la Facultad Multidisciplinaria de Occidente, Universidad de El Salvador. Trabaja como profesor en el Departamento de Medicina de la Facultad Multidisciplinaria Oriental, Universidad de El Salvador. Ha realizado investigaciones con estudiantes de licenciatura en la línea de Enfermedad Renal.

Conflicto de intereses

Declara no tener ningún conflicto de intereses.

Declaración de consentimiento informado

El estudio se realizó, respetando el Código de ética y buenas prácticas editoriales de publicación.

Derechos de uso

Copyright $\odot 2022$ por Jorge Pastor Fuentes Cabrera

Este texto está protegido por una licencia Creative Commons 4.0. Internacional

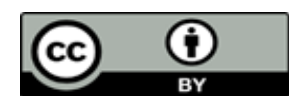

Usted es libre para compartir, copiar y redistribuir el material en cualquier medio o formato y adaptar el documento, remezclar, transformar y crear a partir del material para cualquier propósito, incluso comercialmente, siempre que cumpla la condición de atribución: usted debe reconocer el crédito de una obra de manera adecuada, proporcionar un enlace a la licencia, e indicar si se han realizado cambios. Puede hacerlo en cualquier forma razonable, pero no de forma tal que sugiera que tiene el apoyo del licenciante o lo recibe por el uso que hace. 